International Journal of Biomedicine | June 2021 - Volume 11, Issue Suppl_1: Abstracts from the Third Russian International Conference "Cryo-electron microscopy 2021: achievements and prospects"

\author{
POSTER ABSTRACT PRESENTATIONS
}

SESSION TITLE: EM RESEARCH RELATED TO MEDICINE

DOI: 10.21103/JJBM.11.Suppl_1.P38

\title{
Abstract P-38: Tunable Soft Networks of Wormlike Micelles and Clay Particles
}

Vyacheslav S. Molchanov $^{1}$, Anton S. Orekhov ${ }^{2,3}$, Natalia A. Arkharova ${ }^{4}$, Alexandr I. Kuklin ${ }^{5}$, Andrei V. Rogachev ${ }^{5}$, Olga E. Philippova ${ }^{1}$

${ }^{1}$ Department of Physics, Lomonosov Moscow State University, Moscow, Russia ${ }^{2}$ National Research Centre "Kurchatov Institute," Moscow, Russia ${ }^{3}$ Moscow Institute of Physics and Technology, Dolgoprudny, Russia ${ }^{4}$ FSRC "Crystallography and Photonics" RAS, Moscow, Russia ${ }^{5}$ Joint Institute for Nuclear Research, Dubna, Russia

Background: Over the past few decades, there has been a great deal of interest in the aqueous self-assembly of surfactant molecules into giant wormlike micelles (WLMs). These cylindrical aggregates undergo reversible breakdown processes and in favorable cases can grow up to few tens of micrometers that is comparable with the length of high molecular weight polymer. The viscoelastic properties of WLMs can be easily modified by different additives like salts or polymers. A new emerging research area consists of tuning the WLM solution properties by inorganic nanoparticles. It suggests, in particular, the use of networks of entangled WLMs as a matrix for producing soft nanocomposites with different kinds of embedded nanoparticles that are promising for controlled release, template synthesis, and oilfield applications. These materials can combine adaptive rheological properties of the WLM matrix and the functionality of nanofiller.

Methods: Rheometry and cryo-transmission electron microscopy were combined to investigate the structure and properties of mixed WLMs of zwitterionic oleylamidopropyl dimethyl betaine and anionic sodium dodecyl sulfate surfactants and platelike particles of bentonite clay.

Results: This system demonstrates the formation of giant linear long-lived WLMs, which even at extremely low surfactant concentrations reach a sufficient length to entangle with each other and form a temporally persistent network. The stability of these micelles can be due to electrostatic attraction between the 
headgroups of the anionic and zwitterionic surfactants and favorable volume/length hydrophobic ratio in the surfactant mixture. At increasing surfactant concentration, the long-lived linear micelles transform into fastbreaking branched micelles.

Stable viscoelastic suspensions of clay particles in semi-dilute solutions of WLM were elaborated. They represent a novel type of soft nanocomposite with the tunable matrix. Structural studies revealed that the clay is dispersed in a dense network of entangled WLM in the form of 100-nm tactoids. Rheological investigations demonstrated that clay particles can induce an increase of viscosity and relaxation time by up to one order of magnitude. The effect of the clay becomes more pronounced with increasing content of anionic surfactant, when the micelles become branched. This behavior was explained by the stabilization of micelle-nanoclay junction points due to the screening of the repulsion between positively charged fragments of zwitterionic head groups by added anionic surfactant.

Conclusion: The pronounced effect of nanoparticles on the viscoelasticity of the network formed by branched WLMs was observed for the first time. The nanoparticles-WLM junctions were confirmed by cryo-TEM data. The elaborated systems are of interest for many industrial applications.

Key Words: Wormlike micelles • clay nanoparticles • zwitterionic surfactant

This work was supported by the Russian Science Foundation (project № 1713-01535)

*Corresponding author: Vyacheslav Molchanov. E-mail: molchan@polly.phys.msu.ru

International Journal of Biomedicine. 2021;11 Suppl 1: S28-29

doi: 10.21103/IJBM.11.Suppl_1.P38

(C)2021 International Medical Research and Development Corporation 